\title{
Thermodynamic States of Classical Systems
}

\author{
WILLIAM GREENBERG * \\ Department of Mathematics, Indiana University, Bloomington, Indiana
}

Received April 30, 1971

\begin{abstract}
The existence of a unique thermodynamic state for dilute classical systems is proved for a class of regular multi-particle potentials. The method relies on integral equations for "modified" correlation functions.

Integral equations have been used successfully to prove the existence of equilibrium states of lattice systems defined by multi-particle potentials for both classical and quantum statistical mechanics [1-3], and of classical continuous systems with two-body potentials [4]. However, difficulty in deriving and solving integral equations for continuous systems with any additional multi-particle potentials has led to a belief that these linear methods do not generalize in a natural way to the case of multi-particle potentials in the continuum.

In this paper, we will demonstrate that for stable, multi-particle potentials satisfying a regularity condition, Definition 2.1, the KirkwoodSalzburg equations for the correlation functions generalize to a set of integral equations having, for sufficiently high temperatures, a unique solution which is analytic in its parameters and to which the finite volume correlation functions of the Gibbs ensemble converge. For finite type potentials, a more transparent formulation of regularity is derived. We wish to emphasize that the success in deriving integral equations for multi-particle potentials appears to arise from an optimal use of the expected thermodynamic behavior of the solutions.
\end{abstract}

\section{Integral Equations for Modified Correlation Functions}

We shall study classical continuous systems of point particles in $v$-dimensional Euclidean space, $\mathbb{E}^{v}$. We assume that the potential energy of the particles is specified by a sequence $\left\{\varphi^{m}\right\}_{m \geqq 2}$ of $m$-body potentials; namely, for $n \geqq 2$ and $x_{1}, \ldots, x_{n} \in \mathbb{E}^{v}$, then the potential energy of $n$

* Supported in part by U.S. Air Force Office of Scientific Research Contract No. 53-296-08. 
particles located at $x_{1}, \ldots, x_{n}$ is $U\left(x_{1}, \ldots, x_{n}\right)$, where

$$
U\left(x_{1}, \ldots, x_{n}\right)=\sum_{m=2}^{n} \sum_{1 \leqq i_{1}<i_{2}<\cdots<i_{m} \leqq n} \varphi^{m}\left(x_{i_{1}}, \ldots, x_{i_{m}}\right) .
$$

To simplify notation, we will generally use upper case letters to denote finite subsets of $\mathbb{E}^{v}$ and drop the integer superscripts on the potentials $\varphi$. In this notation,

$$
U(X)=\sum_{T \subset X} \varphi(T)
$$

Where it is desirable to indicate an $n$-element subset of $\mathbb{E}^{v}$, we will write $X_{n}$ or $N(X)=n$, and we will abbreviate the integration symbol $\int_{\Lambda^{n}} d x_{1} \ldots d x_{n}$ by $\int_{\Lambda^{n}} d X_{n}$.

Definition 1.1. The potential $\varphi$ is stable [5] if there exists a positive constant $B$ such that

$$
U(X) \geqq-N(X) B
$$

for all $X \subset \mathbb{E}^{\nu}$. We shall call $\varphi$ ultra-stable if, for some $\mu, 0<\mu<1, B$ can be chosen so that

$$
U(X \cup Y \cup z)-\mu(U(X \cup z)-U(X)) \geqq-N(X \cup Y) B
$$

for all $X, Y, z \subset \mathbb{E}^{v}$ with $N(z)=1$.

For $X \subset \mathbb{E}^{v}$ and any $x \in X$, define

$$
W^{x}(X)=\sum_{S \subset X-x} \varphi(x \cup S)=U(X)-U(X-x)
$$

and let $\omega(X)$ be the element of $X$ which is the smallest element, in some lexicographic order, of the set $\left\{x \in X \mid W^{x}(X) \geqq W^{y}(X), \forall y \in X\right\}$. Then, for any $Y \subset \mathbb{E}^{v}$, define

$$
\begin{aligned}
W(X) & =W^{\omega(X)}(X) \\
W(X, Y) & =\sum_{S \subset X-\omega(X)} \varphi(S \cup Y \cup \omega(X)) .
\end{aligned}
$$

The finite volume correlation functions $\varrho_{\Lambda}$, for $\Lambda$ a bounded, Lebesguemeasurable subset of $\mathbb{E}^{v}$, are given on $E=\bigcup_{n}\left(\mathbb{E}^{v}\right)^{n}$ by

$$
\varrho_{\Lambda}(X)=Z_{\Lambda}^{-1} \chi_{\Lambda}(X) \sum_{n=0}^{\infty} \frac{z^{n+N(X)}}{n !} \int_{\Lambda^{n}} d Y_{n} e^{-\beta U\left(X \cup Y_{n}\right)}
$$


where $\chi_{A}\left(X_{n}\right)$ for each $n \geqq 1$ is the characteristic function of the region $\Lambda^{n} C E$, and $Z_{A}$ is the grand canonical partition function:

$$
Z_{A}=\sum_{n=0}^{\infty} \frac{z^{n}}{n !} \int_{\Lambda^{n}} d Y_{n} e^{-\beta U\left(Y_{n}\right)}
$$

Define modified correlation functions $g_{A}^{\mu}$ for $0<\mu<1$ by

$$
g_{\Lambda}^{\mu}(X)=e^{\mu \beta W(X)} \varrho_{\Lambda}(X) .
$$

The modification factor in these functions will avoid the high energy divergence of the Kirkwood-Salzburg equations for multi-particle potentials.

Theorem 1.2. For $\Lambda$ a bounded Lebesgue-measurable subset of $\mathbb{E}^{v}$ and $\left\{\varphi^{m}\right\}_{m \geqq 2}$ an ultra-stable potential, the finite volume modified correlation functions $g_{A}^{\mu}, 0<\mu<1$, satisfy the integral equations

$$
g_{\Lambda}^{\mu}(X)=\sum_{n=0}^{\infty} \int_{\left(\mathbb{E}^{\nu}\right)^{n}} \frac{d R_{n}}{n !} g_{\Lambda}^{\mu}\left(X \cup R_{n}-\omega(X)\right) K_{\Lambda}^{\mu}\left(X, R_{n}\right)+\chi_{\Lambda}(X) \alpha(X)
$$

with the kernel $K_{\Lambda}^{\mu}$ defined by

$$
\begin{aligned}
& K_{\Lambda}^{\mu}\left(X, R_{n}\right) \\
& =(-1)^{n} z \chi_{\Lambda}(X) e^{\mu \beta W(X)} \sum_{W \subset R_{n}}(-1)^{N(W)} \exp \{-\beta U(X \cup W)+\beta U(X \cup W-\omega(X)) \\
& \left.\quad-\beta \mu W\left(X \cup R_{n}-\omega(X)\right)\right\}
\end{aligned}
$$

and

$$
\alpha(X)=\left\{\begin{array}{ll}
1, & N(X)=0 \\
0, & N(X)>0
\end{array} .\right.
$$

Proof $^{1}$. The identity

$$
z^{N(A)} e^{-\beta U(A)}=Z_{\Lambda} \sum_{m=0}^{\infty} \int_{\Lambda^{m}} \frac{a \cdot m}{m !}(-1)^{m} \varrho_{\Lambda}\left(A \cup V_{m}\right)
$$

for $A \subset \Lambda$ follows from stability, since

$$
\begin{aligned}
& \sum_{n=0}^{\infty} \int_{\Lambda^{n}} \frac{d V_{n}}{n !}(-1)^{n} \sum_{m=0}^{\infty} \int_{\Lambda^{m}} \frac{d S_{m}}{m !} e^{-\beta U\left(A \cup V_{n} \cup S_{m}\right)} \\
= & \sum_{m=0}^{\infty} \int_{\Lambda^{m}} \frac{d R_{m}}{m !} e^{-\beta U\left(A \cup R_{m}\right)} \sum_{\Lambda \subset R_{m}}(-1)^{N(V)}=e^{-\beta U(A)}
\end{aligned}
$$
Marseille. 
and the integrands are absolutely summable for stable potentials. Substitute (3) in (1) to obtain

$$
\begin{aligned}
g_{\Lambda}^{\mu}(X)= & Z_{\Lambda}^{-1} \chi_{\Lambda}(X) e^{\mu \beta W(X)} \sum_{n=0}^{\infty} \frac{z^{n+N(X)}}{n !} \int_{A^{n}} d S_{n} e^{-\beta U\left(X \cup S_{n}-\omega(X)\right)} \\
& \times\left\{e^{-\beta U\left(X \cup S_{n}\right)+\beta U\left(X \cup S_{n}-\omega(X)\right.}\right\} \\
= & \sum_{n=0}^{\infty} \sum_{m=0}^{\infty} \chi_{\Lambda}(X) z \int_{\Lambda^{n}} \frac{d S_{n}}{n !} \int_{\Lambda^{m}} \frac{d V_{m}}{m !} g_{\Lambda}^{\mu}\left(X \cup S_{n} \cup V_{m}-\omega(X)\right) \\
& \times\left\{(-1)^{m} e^{\mu \beta\left(W(X)-W\left(X \cup S_{n} \cup V_{m}-\omega(X)\right)\right.} e^{-\beta U\left(X \cup S_{n}\right)+\beta U\left(X \cup S_{n}-\omega(X)\right)}\right\} \\
= & \sum_{n=0}^{\infty} \int_{\Lambda^{n}} \frac{d R_{n}}{n !} g_{\Lambda}^{\mu}\left(X \cup R_{n}-\omega(X)\right)\left\{\chi_{\Lambda}(X) z \sum_{V \subset R_{n}}(-1)^{N(V)} e^{\mu \beta W(X)}\right. \\
& \left.\times e^{-\beta U\left(X \cup\left(R_{n}-V\right)\right)+\beta U\left(X \cup\left(R_{n}-V\right)-\omega(X)\right)-\mu \beta W\left(X \cup R_{n}-\omega(X)\right)}\right\} .
\end{aligned}
$$

Finally, observe that the $R_{n}$ integration can be extended to $\left(\mathbb{E}^{v}\right)^{n}$, since the $g_{A}^{\mu}$ vanish outside $\Lambda$.

\section{Regular Potentials}

In order for the integral equations to have a unique solution in the limit $\Lambda \rightarrow \infty$, it will be necessary to restrict attention to a class of ultrastable potentials satisfying a regularity requirement.

Definition 2.1. The potential $\varphi$ is regular if the following properties are satisfied for some $\mu, 0<\mu<1$, and for $\beta$ sufficiently small:

(a) Except for $X$ in a set of Lebesgue-measure zero, if $X \subset \mathbb{E}^{v}$ and $W(X)<\infty$, then for every bounded, measurable region $\Lambda \subset \mathbb{E}^{v}, n>0$, and non-degenerate covering $S_{i}, \ldots, S_{j}$ of the set $Y=\left\{y_{1}, \ldots, y_{n}\right\}$, (i.e., $\bigcup_{i=1}^{j} S_{i}=Y, \emptyset \neq S_{i} \neq S_{k}$ for $\left.i \neq k\right)$ the function $e^{-\mu \beta W\left(X \cup Y_{n}-\omega(X)\right)}$ $\times \prod_{i=1}^{j}\left|e^{-\beta W\left(X, S_{2}\right)}-1\right|$ is integrable with respect to $Y$ on $\Lambda$.

(b) Except for $X$ in a set of Lebesgue-measure zero, if $X \subset \mathbb{E}^{v}$ and $W(X)=\infty$, then $W(X, R)>-\infty$ almost everywhere (with respect to $R$ ).

(c) if $\Lambda \rightarrow \infty$ in the sense of van Hove, then

$$
\begin{aligned}
& \lim _{\Lambda \rightarrow \infty} \operatorname{esssup}_{X \subset A}\left\{\sum_{n=1}^{\infty} \int \frac{d Y_{n}}{n !} \sum_{j=1}^{\infty} \sum_{\begin{array}{c}
j_{1}, \ldots, S_{j} \subset Y_{n} \\
S_{i} S_{2}=Y_{n} \\
\emptyset \neq S_{i} \neq S_{k}
\end{array}} e^{-\mu \beta W\left(X \cup Y_{n}-\omega(X)\right)}\right. \\
& \left.\times \prod_{i=1}^{j}\left|e^{-\beta W\left(X, S_{\imath}\right)}-1\right| e^{-\beta(1-\mu) W(X)}\right\}<\infty .
\end{aligned}
$$


We shall see that for an ultra-stable, regular potential, a unique infinite volume state can be defined for dilute systems. An equivalent formulation of regularity which gives a more transparent restriction on the potentials $\varphi$ or the "interaction energies" $W$ would obviously be desirable. Although we cannot derive this in general, we will give in Section 3 a sufficient criterion for regularity for a class of physically reasonable potentials.

Ultra-stability yields immediately.

Lemma 2.2. For $z$ sufficiently small, $g_{A}^{\mu} \in L^{\infty}(E)$, the Banach space of bounded, Lebesgue-measurable functions on $E$ with sup norm \|\|$_{\infty}$.

Lemma 2.3. If $\varphi$ is an ultra-stable, regular potential, then the kernel $K_{\Lambda}^{\mu}$ is a bounded operator on $L^{\infty}(E)$, with norm

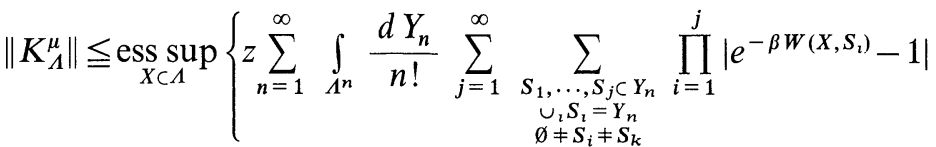

$$
\begin{aligned}
& \times \exp \left\{-\beta(1-\mu) W(X)-\mu \beta W\left(X \cup Y_{n}-\omega(X)\right)\right\} \text {. }
\end{aligned}
$$

$$
\begin{aligned}
& K_{\Lambda}^{\mu}(X, R) \exp \{\beta(1-\mu) W(X)+\beta \mu W(X \cup R-\omega(X))\} \\
& =z \chi_{\Lambda}(X)(-1)^{N(R)} \sum_{W \subset R}(-1)^{N(W)} \exp \left\{-\beta \sum_{\substack{S \subset W \\
S \neq \emptyset}} W(X, S)\right\} \\
& =z \chi_{\Lambda}(X)(-1)^{N(R)} \sum_{W \subset R}(-1)^{N(W)} \sum_{j=1}^{\infty} \sum_{\substack{S_{1}, \ldots, S_{j} \subset W \\
\emptyset \neq S_{\imath} \neq S_{k}}} \prod_{i=1}^{j}\left(e^{-\beta W\left(X, S_{\imath}\right)}-1\right) \\
& =z \chi_{\Lambda}(X) \sum_{j=1}^{\infty} \sum_{\substack{S_{1}, \ldots, S_{j} \subset R \\
\emptyset \neq S_{i} \neq S_{k}}} \sum_{V \subset R-\cup_{i} S_{i}}(-1)^{N(V)} \prod_{i=1}^{j}\left(e^{-\beta W\left(X, S_{i}\right)}-1\right) \\
& =z \chi_{\Lambda}(X) \sum_{j=1}^{\infty} \sum_{\substack{S_{1}, \ldots, S_{j} \subset R \\
S_{i} S_{i}=R \\
\emptyset \neq S_{l} \neq S_{k}}} \prod_{i=1}^{j}\left(e^{-\beta W\left(X, S_{i}\right)}-1\right)
\end{aligned}
$$

observing in the last equation that $\sum_{V \subset T}(-1)^{N(V)}$ vanishes unless $T=\emptyset$.

Lemma 2.4. If $\varphi$ is an ultra-stable, regular potential and $\beta$ is sufficiently small, then for some $\mu<1$, the $K_{\Lambda}^{\mu}$ converge to a bounded operator $K_{\infty}^{\mu}$ on $L^{\infty}(E)$ as $\Lambda \rightarrow \infty$ in the following sense. For every sequence $\left\{\Lambda_{i}\right\}$ 
W. Greenberg:

which converges $\lim _{i \rightarrow \infty} \Lambda_{i}=\infty$ in the sense of van Hove, and for each $f \in L^{1}(E)$, $K_{\Lambda_{2}}^{\mu+} f \rightarrow K_{\infty}^{\mu+} f$ strongly in $L^{1}(E)$.

Here, and throughout, $T^{+}$, for $T$ an operator on $L^{\infty}(E)$, signifies the adjoint of T restricted to the space $L^{1}(E)$ of absolutely Lebesgue-integrable functions with norm \|\|$_{1}$.

Proof. Define $K_{\infty}^{\mu}$ pointwise by Eq. (2) with the characteristic function $\chi_{\Lambda}$ replaced by the identity function,

$$
K_{\Lambda}^{\mu}(X, Y)=\chi_{\Lambda}(X) K_{\infty}^{\mu}(X, Y)
$$

and let $\sigma(X)$ denote the Lebesgue measure of any set $S \subset E$.

If $\varepsilon>0$ and $\sigma(C)<\infty$, there exists a bounded set $C^{\prime} C C$ with $\sigma\left(C-C^{\prime}\right)<\varepsilon$. For $i$ sufficiently large, $\Lambda_{i} \supset C^{\prime}$, and then

$$
K_{\Lambda_{r}}^{\mu+} f(s)=\chi_{\Lambda_{i}}(s) K_{\infty}^{\mu+} f(s)=K_{\infty}^{\mu+} f(s)
$$

if $s \in C^{\prime}$. Thus

$$
\sigma\left(\left\{s \mid s \in C, K_{\Lambda_{\imath}}^{\mu+} f(s)-K_{\infty}^{\mu+} f(s) \neq 0\right\}\right)<\varepsilon .
$$

That is, $K_{\Lambda_{i}}^{\mu+} f \rightarrow K_{\infty}^{\mu+} f$ in Lebesgue measure on every measurable set of finite measure.

Furthermore, $K_{\Lambda_{2}}^{\mu+} f \rightarrow K_{\infty}^{\mu+} f$ weakly. For if $\varepsilon>0$, choose a bounded measurable region $D \subset E$ so that $\left\|f-f_{D}\right\|_{1}<\varepsilon$, where $f_{D}$ denotes the restriction of $f$ to $D$. Then, for $i$ sufficiently large, $\Lambda_{i} \supset D$, and for each $\tau \in L^{\infty}(E)$,

$$
\begin{aligned}
\left|\int_{E} f(\bar{X})\left(\left(K_{\Lambda_{i}}^{\mu}-K_{\infty}^{\mu}\right) \tau\right)(X) d X\right| \leqq\left|\int_{D} f(\bar{X})\left(\left(K_{\Lambda_{i}}^{\mu}-K_{\infty}^{\mu}\right) \tau\right)(X) d X\right| \\
+\varepsilon\left\|K_{\Lambda_{l}}^{\mu}-K_{\infty}^{\mu}\right\|\|\tau\|_{\infty} \leqq \varepsilon\left(\left\|K_{\Lambda_{\imath}}^{\mu}\right\|+\left\|K_{\infty}^{\mu}\right\|\right)\|\tau\|_{\infty} .
\end{aligned}
$$

The Lemma follows from the observation that strong convergence in $L^{1}$ is equivalent to weak convergence and convergence in measure on every measurable set of finite measure.

Definition 2.5. Let $g_{\infty}^{\mu}$ be a solution of the integral equation

$$
\left(1-K_{\infty}^{\mu}\right) g_{\infty}^{\mu}=\alpha .
$$

Call $\varrho_{\infty}$, defined by

$$
\varrho_{\infty}(X)=e^{-\beta \mu W(X)} g_{\infty}^{\mu}(X)
$$

an infinite volume state.

When $\left\|K_{A}^{\mu}\right\| \leqq C<1$ for all $\Lambda, K_{\infty}^{\mu}$ is a strict contraction on $L^{\infty}(E)$, and the integral equation in Definition 2.5 has a unique solution. 
Theorem 2.6. If $\varphi$ is an ultra-stable, regular potential, then in a region of the $\beta-z$ plane defined by

$$
\begin{gathered}
\lim _{\Lambda \rightarrow \infty} \operatorname{ess} \sup _{X \subset A}\left\{\sum_{n=1}^{\infty} \int \frac{d Y_{n}}{n !} \sum_{j=1}^{\infty} \sum_{\substack{S_{1}, \ldots, S_{j} \subset Y_{n} \\
\cup_{i} S_{2}=Y_{n} \\
\emptyset \neq S_{i} \neq S_{k}}} \prod_{i=1}^{j}\left|e^{-\beta W\left(X, S_{\imath}\right)}-1\right|\right. \\
\times \exp \left\{-\beta(1-\mu) W(X)-\beta \mu W\left(X \cup Y_{n}-\omega(X)\right)\right\} \\
<z^{-1}
\end{gathered}
$$

there is a unique infinite volume state $\varrho_{\infty}$. If $\Lambda \rightarrow \infty$ in the sense of van Hove, then $g_{\Lambda}^{\mu}$ converges weakly to $g_{\infty}^{\mu}$, and $\varrho_{\Lambda}$ converges pointwise to $\varrho_{\infty}$ almost everywhere. In particular, if $W(X)$ is bounded below, then $\varrho_{\Lambda}$ converges to $\varrho_{\infty}$ weakly.

Proof. It remains only to be proved that for any $f \in L^{1}(E), \varepsilon>0$, and sequence $\left\{\Lambda_{i}\right\}$ with $\Lambda_{i} \rightarrow \infty$,

$$
\left|\left(f,\left(I-K_{\Lambda_{\imath}}^{\mu}\right)^{-1} \alpha \chi_{\Lambda_{\imath}}\right)-\left(f,\left(I-K_{\infty}^{\mu}\right)^{-1} \alpha\right)\right|<\varepsilon
$$

if $i$ is sufficiently large, where we have used $(f, \tau)$ to denote the integral over $E$ of the product $f(X) \tau(X), f \in L^{1}(E), \tau \in L^{\infty}(E)$. Estimating $\left(I-K^{+}\right)^{-1} f$ by $\sum_{j=0}^{N}\left(K^{+}\right)^{j} f$ and $\left(K_{\Lambda}\right)^{i}-(K)^{i}$ by $\sum_{k=1}^{i} K_{\Lambda}^{i-k}\left(K_{\Lambda}-K\right) K^{k-1}$, it is clear that $\left(I-K_{\Lambda_{\imath}}^{\mu+}\right)^{-1} f \rightarrow\left(I-K_{\infty}^{\mu+}\right)^{-1} f$ strongly, by Lemma 2.4 . Then:

$$
\begin{aligned}
& \left|\left(f,\left(I-K_{\Lambda_{i}}^{\mu}\right)^{-1} \alpha \chi_{\Lambda_{i}}\right)-\left(f,\left(I-K_{\infty}^{\mu}\right)^{-1} \alpha\right)\right| \\
& \leqq\left|\left(\left(I-K_{\Lambda_{i}}^{\mu+}\right)^{-1} f-\left(I-K_{\infty}^{\mu+}\right) f, \alpha \chi_{\Lambda_{i}}\right)\right| \\
& +\left|\left(\left(I-K_{\infty}^{\mu+}\right)^{-1} f, \alpha\left(1-\chi_{\Lambda_{i}}\right)\right)\right| \\
& \leqq \varepsilon / 2+\left|\left(\left(I-K_{\infty}^{\mu+}\right) f, \alpha\left(1-\chi_{\Lambda_{\imath}}\right)\right)\right|
\end{aligned}
$$

for $i$ sufficiently large. But if $D \subset E$ is a bounded measurable region satisfying $\left\|\left(I-K_{\infty}^{\mu+}\right)^{-1} f-\left(\left(I-K_{\infty}^{\mu+}\right)^{-1} f\right)_{D}\right\|_{1}<\varepsilon / 2$, then

$$
\left|\left(\left(I-K_{\infty}^{\mu+}\right)^{-1} f, \alpha\left(1-\chi_{\Lambda_{i}}\right)\right)\right|<\varepsilon / 2
$$

when $\Lambda_{i} \supset D$.

Corollary 2.7. If $\varphi$ is an ultra-stable, regular potential, then in the region of the $\beta-z$ plane defined in Theorem $2.6, \varrho_{\infty}(X)$ is an analytic function of $\beta$ and $z$. If $\varphi$ is a translation-invariant potential, then $\varrho_{\infty}$ is translation invariant. 


\section{Potentials of Finite Type}

For potentials which satisfy some physically reasonable restrictions of finiteness and continuity, a sufficient condition for regularity can be given which directly generalizes the usual definition of regularity [5] for two-body interactions.

Toward this end, define $d: E \rightarrow \mathbb{R}$ by $d(X)=\min _{\substack{x, y \in X \\ x \neq y}}|x-y|$. If $\left\{\delta_{n}\right\}_{n \geqq 1}$ is a sequence of non-negative numbers, let $E_{\left\{\delta_{n}\right\}}=\left\{X \in E \mid d(X)>\delta_{N(X)}\right\} \subset E$, and write $I_{X}(Y)$ for $\left|e^{-\beta W(X, Y)}-1\right| / N(Y) !, X, Y \in E$.

Definition 3.1. A potential $\left\{\varphi^{n}\right\}_{n \geqq 2}$ is of finite type if

(a) for each $n, \varphi^{n}$ is continuous on $\left(\mathbb{E}^{v}\right)^{n} \cap E_{\{0\}}$,

(b) $\varphi^{2}(x, y) \rightarrow \infty$ as $d(x \cup y) \rightarrow 0, x, y \in \mathbb{E}^{v}$,

(c) $\varphi$ is bounded below on $E$,

(d) for each $X \in E$, there exists a sequence $\left\{t_{X}^{n}\right\}_{n \geqq 1}$ of step functions in $L^{1}(E)$, with intervals of uniform volume $1 / n$ and bounded below by $I_{X}$, such that $t_{X}^{n} \rightarrow I_{X}$ strongly and $\left\{t_{X}^{n}\right\}_{n \geqq 1}$ is Cauchy uniformly in $X$.

Lemma 3.2. If $\varphi$ is ultra-stable and of finite type, then $W(X) / N(X)$ is bounded below on $E$, and $W(X) \rightarrow \infty$ as $d(X) \rightarrow 0$.

Theorem 3.3. A stable potential $\varphi$ of finite type is regular if for $\beta$ sufficiently small and $\Lambda \rightarrow \infty$ in the sense of van Hove,

$$
\lim _{\Lambda \rightarrow \infty} \sup _{X \subset A}\left\{\sum_{n=1}^{\infty} \int d Y_{n} I_{X}\left(Y_{n}\right)\right\}<\infty .
$$

Proof. Let $f(n)$ be a positive decreasing function of the positive integers $n$, to be fixed later, and choose $\left\{\delta_{n}\right\}_{n \geqq 1}$ to satisfy

$$
e^{-\beta W(Y)}<f(n) \text { whenever } N(Y)=n \text { and } d(Y)<\delta_{n} .
$$

It is clearly sufficient to prove:

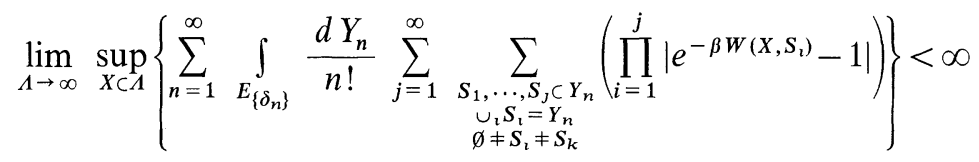

uniformly in $\left\{\delta_{n}\right\}$, and for each $\varepsilon>0$ and $X \in E$,

$$
\left\{\sum_{n=1}^{\infty} \int_{E-E_{\left\{\delta_{n}\right\}}} \frac{d Y_{n}}{n !} \sum_{j=1}^{\infty} \sum_{\substack{S_{1}, \ldots, S_{j} \subset Y_{n} \\ \sigma_{i}, Y_{S}=Y_{n} \\ \emptyset \neq S_{i} \neq S_{k}}}(f(n))^{\mu} \prod_{i=1}^{j}\left|e^{-\beta W\left(X, S_{i}\right)}-1\right|\right\}<\varepsilon
$$

for some $\left\{\delta_{n}\right\}_{n \geqq 1}$.

Consider (6) first. Fix $X \in E$, let $\varepsilon>0$, and assume $\varphi$ is bounded below by $M<-1$. Then $W\left(X, S_{i}\right)>2^{N(X)} M$ and $\left|e^{-\beta W\left(X, S_{i}\right)}-1\right|<e^{2^{N(X)} \beta M}$. 
By (4), $\Lambda_{0}$ can be chosen sufficiently large so that

$\int_{E-\Lambda^{n}} \frac{d Y_{n}}{n !}\left|e^{\beta W\left(X, Y_{n}\right)}-1\right|<\varepsilon$ and $\left|e^{-\beta W\left(X, Y_{n}\right)}-1\right|<1$ if $Y_{n} \cap \Lambda^{n} \neq Y_{n}$

for all $n$ and all $\Lambda \supset \Lambda_{0}$. Split the integration in (6) over the sets $\left(E-E_{\left\{\delta_{n}\right\}}\right) \cap\left(\bigcup_{n} \Lambda_{0}^{n}\right)$ and $\left(E-E_{\left\{\delta_{n}\right\}}\right) \cap\left(E-\bigcup_{n} \Lambda_{0}^{n}\right)$. Since the number of coverings of $Y_{n}$ by $j$ subsets is certainly less than $\left(\begin{array}{c}2^{n} \\ j\end{array}\right)$, the integration over $\left(E-E_{\left\{\delta_{n}\right\}}\right) \cap\left(\bigcup_{n} \Lambda_{0}^{n}\right)$ can be bounded by

$$
\sum_{n=1}^{\infty}\left\{\frac{V\left(\Lambda_{0}\right)^{n}}{n !} 2^{2^{n}} e^{\beta M 2^{N(X)+n}}(f(n))^{\mu}\right\}
$$

and the integration over $\left(E-E_{\left\{\delta_{n}\right\}}\right) \cap\left(E-\bigcup_{n} \Lambda_{0}^{n}\right)$ by $\varepsilon$ times the above. Eq. (6) then is satisfied by choosing $f(n)$ so that (7) is less than $\varepsilon$.

To prove (5), fix $\Lambda$, let $P_{n}=\left\{\left[x_{1}, \ldots, x_{n}\right]\right\}$ be a uniform partition of $\Lambda^{n}$ with $V\left(\left[x_{1}, \ldots, x_{n}\right]\right)=\lambda$, for all $n$; here the intervals $\left[x_{1}, \ldots, x_{n}\right]$ of the partition are labeled by the centers $\left(x_{1}, \ldots, x_{n}\right)$ of the hypercubes $\left[x_{1}, \ldots, x_{n}\right]$. Define

$$
J_{\left\{x_{1} \cup \ldots \cup x_{n}\right\}}(X)=\sup _{S \in\left[x_{1}, \ldots, x_{n}\right]}\left|e^{-\beta W(X, S)}-1\right|
$$

and choose $\lambda$ sufficiently small so that

$$
\left|\sum_{n=1}^{\infty}\left(\sum_{P_{n}} J_{\left\{x_{1} \cup \ldots \cup x_{n}\right\}}(X) \frac{\lambda}{n !}-\int_{\Lambda^{n}} \frac{d Y_{n}}{n !}\left\{\left|e^{-\beta W\left(X, Y_{n}\right)}-1\right|\right\}\right)\right|<\varepsilon
$$

for all $X$. Then:

$$
\begin{aligned}
& \sum_{n=1}^{\infty} \int_{E_{\left\{\delta_{n}\right\}} \cap A^{n}} \frac{d Y_{n}}{n !} \sum_{j=1}^{\infty} \sum_{\substack{S_{1}, \ldots, S_{j} \subset Y_{n} \\
\text {, } Y_{n}, Y_{n} \\
\emptyset \neq S_{\imath} \neq S_{k}}} \prod_{i=1}^{j}\left|e^{-\beta W\left(X, S_{2}\right)}-1\right| \\
& \leqq \sum_{n=1}^{\infty} \sum_{P_{n}} \frac{\lambda}{n !} \sum_{j=1}^{\infty} \sum_{\substack{S_{1}, \ldots, S_{j}<\left\{x_{i} \cup \ldots \cup x_{n}\right\} \\
\dot{b}_{i} S_{2}=Y_{n} \\
\emptyset \neq S_{l} \neq S_{k}}}\left(\prod_{i=1}^{j} J_{\left\{S_{i}\right\}}(X) \lambda\right) \lambda^{-j} \\
& \leqq \sum_{j=1}^{\infty} \frac{1}{j !}\left\{\sum_{n=1}^{\infty} \frac{1}{n ! \lambda} \sum_{P_{n}} J_{\left\{x_{1} \cup \ldots \cup x_{n}\right\}}(X) \lambda\right\}^{j} \\
& \leqq \exp \left\{\sum_{n=1}^{\infty} \frac{1}{\lambda} \int_{\Lambda^{n}} \frac{d Y_{n}}{n !}\left\{\left|e^{-\beta W\left(X, Y_{n}\right)}-1\right|\right\}+\frac{\varepsilon}{\lambda}\right\}<\infty
\end{aligned}
$$

which completes the proof. 
From the foregoing, it is easy to construct examples of regular multibody potentials. The simplest such example is a translation-invariant potential with two and three body components only, satisfying:

i) $\varphi^{2}(0, x)$ is continuous, bounded below, and absolutely integrable outside a set of finite Lebesgue measure, and $\varphi^{2}(0, x) \rightarrow \infty$ as $|x| \rightarrow 0$.

ii) $\varphi^{3}$ is continuous, non-negative, and symmetric in its arguments, and $\varphi^{3}\left(0, x_{1}, x_{2}\right)$ vanishes outside a bounded region.

\section{Integral Equation Techniques}

The technique of using integral equations to study families of functions on a Banach space is most fruitful if either the Banach space is chosen to accomodate the special properties of the family, as in Ruelle's analysis of two-body potentials [5], or if, as above, the family is optimally modified to fit a fixed Bánach space. In either case, one seeks a priori bounds for the correlation functions, and thus expects for thermodynamic interactions implicit cutoffs at high particle densities and high energy densities. It is for this reason that it is natural to study the modified correlation functions with their "modified" high energy behavior rather than the correlation functions themselves.

Acknowledgement. I am indebted to Professor Visconti and the Centre de Physique Theorique of the Centre National de la Recherche Scientifique for their kind hospitality and support.

\section{References}

1. Gallavotti, G., Miracle-Sole, S.: Commun. math. Phys. 7, 274 (1968).

2. - - Robinson, D.: Phys. Letters 25 A, 493 (1967).

3. Greenberg, W.: Commun. math. Phys. 13, 335 (1969).

4. Ruelle, D.: Ann. Phys. 25, 109 (1963).

5. - Statistical mechanics. New York: W. A. Benjamin 1969.

W. Greenberg

Dept. of Mathematics

Indiana University

Bloomington, Indiana 47401, USA 